\title{
Three component cartilage framework reconstruction for correction of post-traumatic nasal septal collapse
}

\author{
Uday Bhat, Tarush Gupta, Mahesh Nair, Mayur Mantri, Mangesh Pawar, Amresh Baliarsing \\ Department of Plastic Surgery, Topiwala National Medical College and B.Y.L. Nair Charitable Hospital, Mumbai, Maharashtra, \\ India
}

Address for correspondence: Dr. Tarush Gupta, House No. 113, N-Area, Near Airport, Chandigarh - 160 003, India. E-mail: tarush139@gmail.com

\section{ABSTRACT}

Background: Post-traumatic nasal septal collapse results in flat, broad, non-projecting nose due to mid-vault collapse. These patients may have airway obstruction due to poorly supported internal valve. Traditional techniques like 'cantilever graft technique' or conventional 'L-graft technique' produce a rigid lobule as the single unit framework extends to the tip. These grafts also lack in internal valve support. Materials and Methods: Twelve patients with post-traumatic nasal septal collapse were treated with three component cartilage framework technique in the past 3 years. The framework was reconstructed in three components-septal, columellar and dorsal onlay; using costal cartilage. If needed, upper lateral cartilage support grafts were also provided. Results: At 6 months' follow-up, all 12 patients were satisfied with the outcome. The nasal projection and dorsal definition were aesthetically pleasing, and there was free natural movement of the lobular part of the nose. One graft was revised for further improvement in outcome. Airway obstruction, when present was also relieved. Conclusion: This technique has distinct advantages over the conventional techniques as the framework of separate components maintains pliability of the lobule, supports the internal valve and offers good control of aesthetic needs.

\section{KEY WORDS}

Cartilage reconstruction; collapse; nasal deformity; rhinoplasty; septal; trauma

\section{INTRODUCTION}

ost-traumatic nasal septal collapse is usually a consequence of a direct blow to the nose or vehicular accident. Deformity occurs due to compression injury to the cartilaginous part of the septum, and if not adequately treated, may lead to flat, broad, non-projecting

\begin{tabular}{|l|l|}
\hline \multicolumn{2}{|c|}{ Access this article online } \\
\hline Quick Response Code: & Website: \\
\hline & www.ijps.org \\
\cline { 2 - 2 } & DOI: \\
\hline
\end{tabular}

nose due to collapse of the mid-vault. The loss of cartilage volume is attributed to the septal haematoma with or without infection. ${ }^{[1]}$ They may have airway obstruction as the internal valve is not well supported. Conventionally, these injuries have been treated either with 'cantilever graft technique' ${ }^{\mid 2]}$ or conventional 'L-graft' technique. ${ }^{[3]}$ In

This is an open access article distributed under the terms of the Creative Commons Attribution-NonCommercial-ShareAlike 3.0 License, which allows others to remix, tweak, and build upon the work non-commercially, as long as the author is credited and the new creations are licensed under the identical terms.

For reprints contact: reprints@medknow.com

How to cite this article: Bhat U, Gupta T, Nair M, Mantri M, Pawar M, Baliarsing A. Three component cartilage framework reconstruction for correction of post-traumatic nasal septal collapse. Indian J Plast Surg 2017;50:236-43. 
both these techniques, the monoblock framework extends to the tip, resulting in a rigid lobule. Second, these grafts do not provide adequate support to the internal valve and may not relieve airway obstruction. In addition, the cantilever graft also adds stress to the framework, skin and soft tissue..$^{[4,5]}$

Daniel ${ }^{[6]}$ has recommended composite reconstruction of the framework with cartilage grafts. We have modified the method described by Daniel in the Indian context and have performed correction of post-traumatic nasal septal collapse by a three-component framework consisting of a septal component, a columellar component and a solid dorsal onlay component. This technique has three distinct advantages: preservation of the natural pliability of the lobule, improvement of airway subsequent to the internal valve support and better aesthetic result because of the dorsal definition offered by the onlay graft.

\section{Description of the deformity}

Constantian $^{[7]}$ has given a detailed description of the deformity following septal collapse. The characteristic features include flattening of the nasal dorsum with accentuation or development of a dorsal hump and narrowing of the middle third of the nose with internal valvular incompetence. There may be a change in the nasal length, the apparent increase in the nasal base size and apparent bony vault widening. Loss of caudal septal support may result in sharpening of the subnasale, upper lip retrusion with flattening and posterior displacement of the upper lip. On septal support test (Daniel), ${ }^{[6]}$ compression of the lobule offers no resistance [Figure 1].

All the above features may not be uniformly present in post-traumatic deformities. However, in the frontal view, broad and flat appearance of the nose is obvious [Figure 2]. In the lateral view, the tip appears blunt, and there is hollowness in the supratip area because of collapse of critical part (dorsocaudal strut) of the septum [Figure 3].

We have noticed two additional features of the deformity:

1. Shadow sign: A horizontal linear shadow is usually seen in the middle of the defect and when present, is pathognomonic of septal collapse that leads to the deformity [Figure 4a]

2. Tip touch sign: On applying gentle pressure on the nasal tip the edges of the pyriform aperture may be visible [Figure $4 \mathrm{~b}$ ].

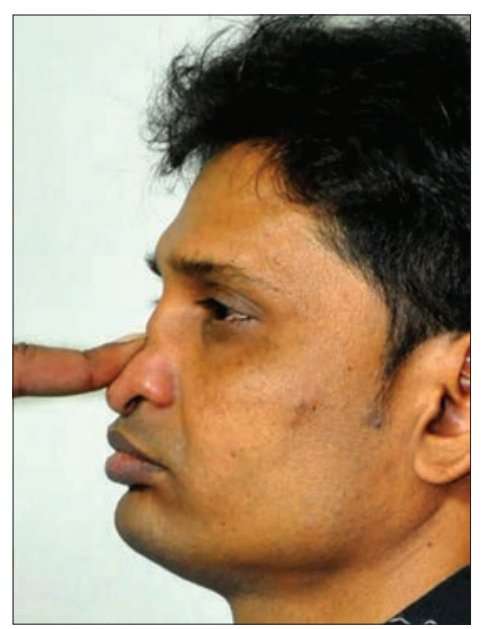

Figure 1: Septal support test - there is no resistance to the digital pressure applied to the supratip area

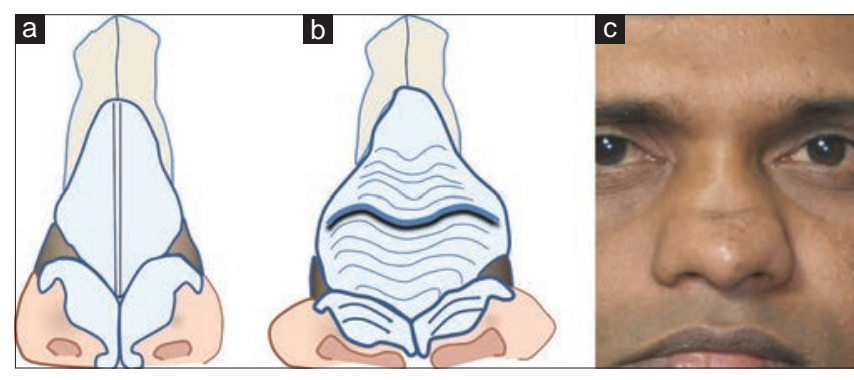

Figure 2: Appearance of nasal dorsum in frontal view - (a) Normal architecture (b) architecture in septal collapse (c) typical deformity with a flat and broad nose

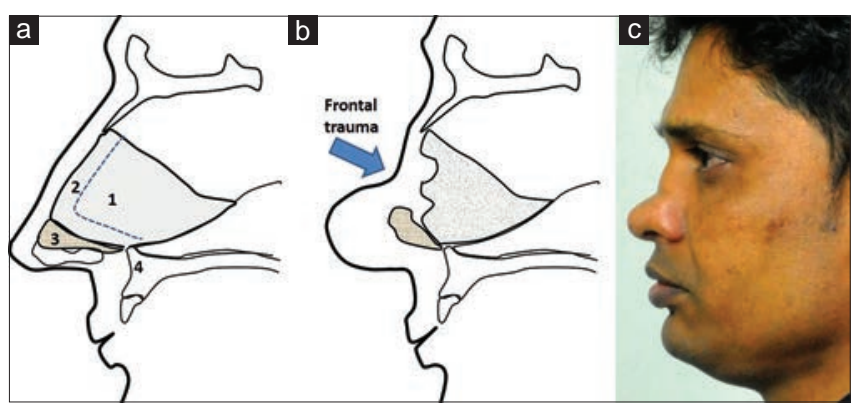

Figure 3: (a) Normal architecture of the nose. 1: Quadrangular septal cartilage 2: Critical part of the septum 3: Membranous septum 4: Anterior nasal spine (b and c) Septal collapse resulting in loss of critical part of septum and subsequent blunt tip and hollowness of the supratip area

The airway obstruction in these patients is usually due to lack of support to the internal valve and not because of deviation of residual septum.

\section{MATERIALS AND METHODS}

In a retrospective study, 12 patients with post-traumatic nasal septal collapse were treated with this technique in the past 3 years (May 2013-May 2016). All patients had a history of trauma resulting in the deformity. The details of the primary injury and subsequent treatment 


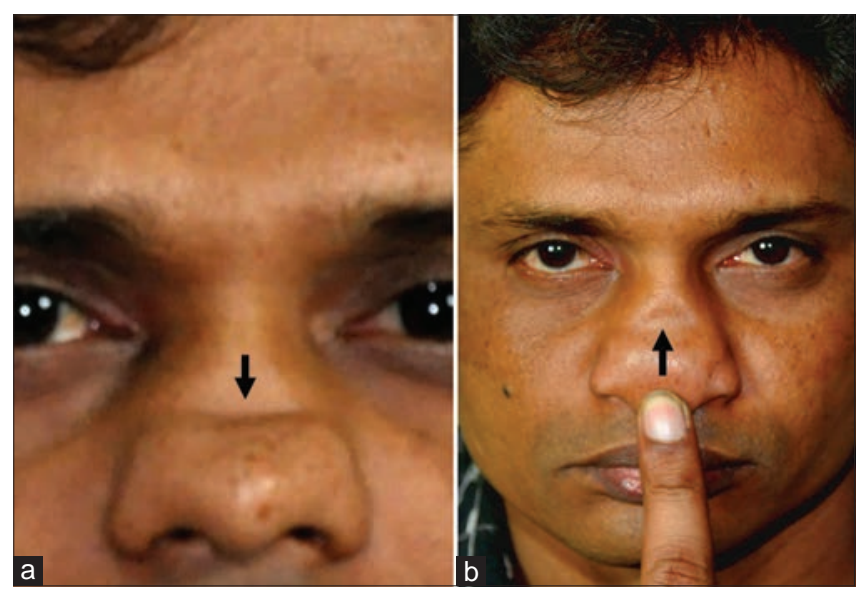

Figure 4: Clinical signs. (a) The shadow sign- The arrow shows the characteristic horizontal shadow. (b) Tip touch sign - A gentle pressure applied to the tip makes the edges of pyriform aperture distinct (arrow)

were not known as none of them were treated by us for the primary injury and presented to us a few years later for correction of the resultant deformity. Ten were males and two were females. The youngest patient was 20 years old and the eldest being 32 years old, with a mean age of 26 years. Two males and one female had airway obstruction. The Cottle's test in these patients was positive indicating internal valve collapse. Anterior rhinoscopy in all patients showed no abnormality, and none of the patients had a deviation of residual septum. The computed tomography scans of the patients with airway obstruction showed no anatomical abnormality.

\section{Technique of correction}

Construction of the new framework requires a largevolume of cartilage. Only costal cartilage can provide such a large volume and hence was used as donor tissue. Either the seventh, eighth or both costal cartilages were harvested and divided to obtain multiple pieces [Figure 5a]. Cutting and carving of the cartilage was done using the precision carving technique. ${ }^{[8]}$

The framework was reconstructed in three components.

1. Septal construct: Restores the structurally important dorsal and caudal septal strips (dorsocaudal strut). It was made with three pieces:

a. A pair of grafts, 20-25 mm long, 10-12 mm wide and $3 \mathrm{~mm}$ thick, serving as the dorsal septal strip. The combined thickness of the bi-lamellar dorsal strip is akin to the spreader grafts and supports the internal valve

b. A graft $20-25 \mathrm{~mm}$ long, $10-12 \mathrm{~mm}$ wide and 2-3 mm thick, serving as the caudal septal strip.

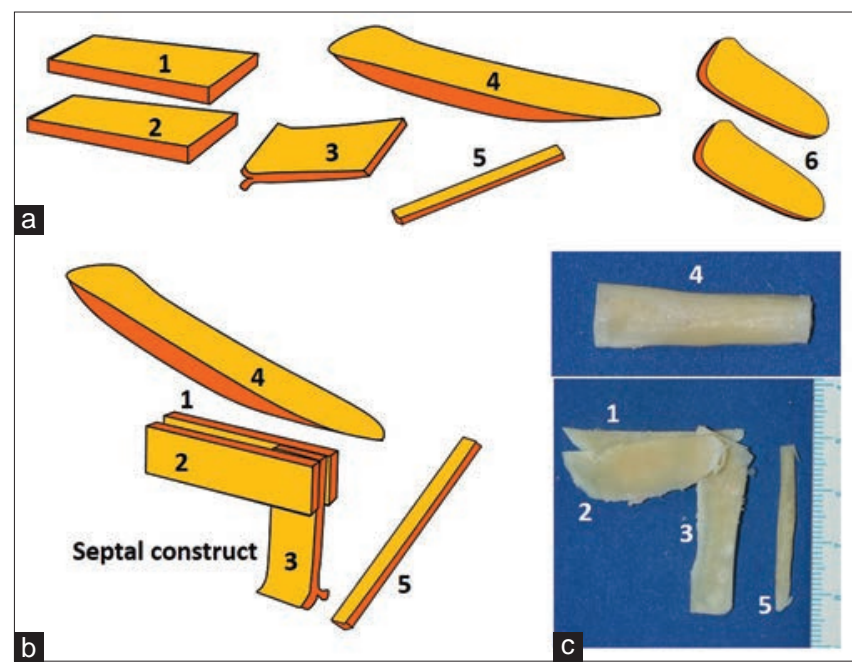

Figure 5: Framework construction (a) multiple pieces carved from costal cartilage. 1 and 2: Dorsal strips. 3: Caudal strip. 4: Dorsal onlay graft. 5: Columellar strut. 6: Lateral cartilage support grafts. Additional pieces may be required for contouring elsewhere in the nose. (b and c). The assembly of three component framework: the septal construct is made from pieces 1 , 2 and 3: Sutured tongue-in-groove at a suitable angle. Piece 4 is for dorsal onlay and piece 5 is for columellar strut

The graft was split at the posterior end for anchoring to the anterior nasal spine (ANS).

The bi-lamellar dorsal strip and the caudal strip were sutured tongue in groove at a desired angle [Figure 5b] with nasal width of approximately $6 \mathrm{~mm}$.

2. Columellar strut: Provides tip support. The graft is cut very thin, approximately $2-3 \mathrm{~mm}$ in thickness so that it does not encroach on the membranous septum

3. Dorsal onlay graft: Helps establish nasal projection and width [Figure $5 \mathrm{~b}$ and $\mathrm{c}$. This graft also contributes to the internal valve support.

Additional pieces needed for contouring or upper lateral cartilage support [Figure 5a] were also obtained if required.

The open approach was used for insertion of the framework in all patients. A midline incision was made on the flat area between the two upper lateral cartilages and two mucoperichondrial leaves (flaps) were opened to create a pocket of adequate size to receive the framework [Figure 6]. The anterior and caudal septal area is devoid of cartilage, and it is technically difficult to separate the two mucoperichondrial leaves to create a pocket. The septal construct was fabricated in situ. The pieces for the septal construct were arranged inside the pocket and were fixed at either ends, to the nasal bones above and ANS below. The tongue in groove fixation sutures were taken last after adjusting the length and 


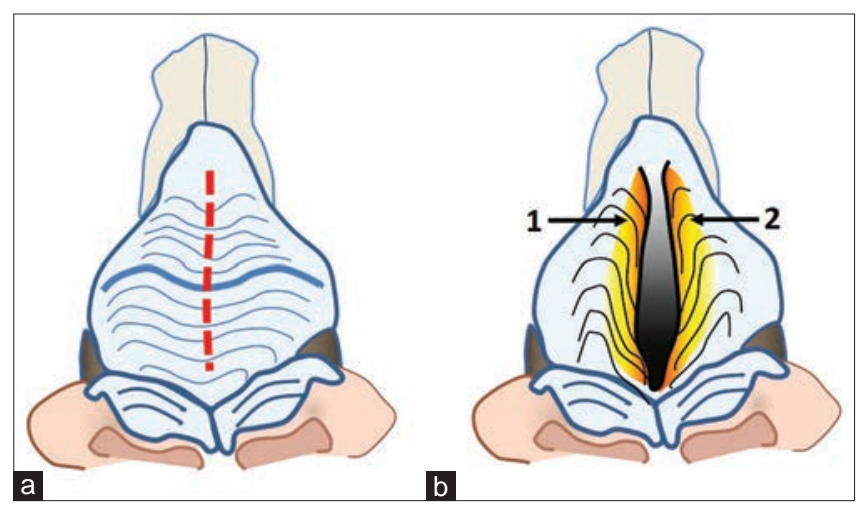

Figure 6: (a) Incision for creation of the pocket. (b) The pocket with the two mucoperiosteal leaves $(1,2)$ reflected

the angle. The sides of the dorsal strips were sutured to the upper lateral cartilages [Figure 7].

Tip support was provided by using a separate columellar strut sutured to the medial crura of alar cartilages and loosely anchored to the soft tissue around ANS [Figure 8]. Dorsal augmentation was done with an onlay graft to achieve the desired projection and definition. The dimensions of the graft were decided by pre-operative nasal analysis using Guyuron's technique ${ }^{[9]}$ and also by filling the defect with a template carved from a soap cake so as to obtain a pleasant shape. If required, the upper lateral cartilages were reinforced with additional support grafts. Osteotomies and alar wedge resection were also done when required.

\section{RESULTS}

All patients were satisfied with outcome as seen on 6 months' follow-up. The three patients with airway obstruction had relief indicating good structural support. The projection of the dorsum as well as the tip and dorsal definition were aesthetically pleasing. Importantly, there was free natural movement of the lobular part of the nose. One patient (patient 2) had the prominence of the dorsal graft, yet she was satisfied with the result. She was offered a revision and this was done 9 months after the first operation with a further improvement in result. To illustrate the technique, following are a few representative cases:

\section{Patient 1}

A 28-year-old man was assaulted with a direct hit to the nose 8 years back. Pathognomonic shadow sign and tip touch signs were present. The three component framework was constructed from eighth costal cartilage and positioned in the anatomical areas. Osteotomies

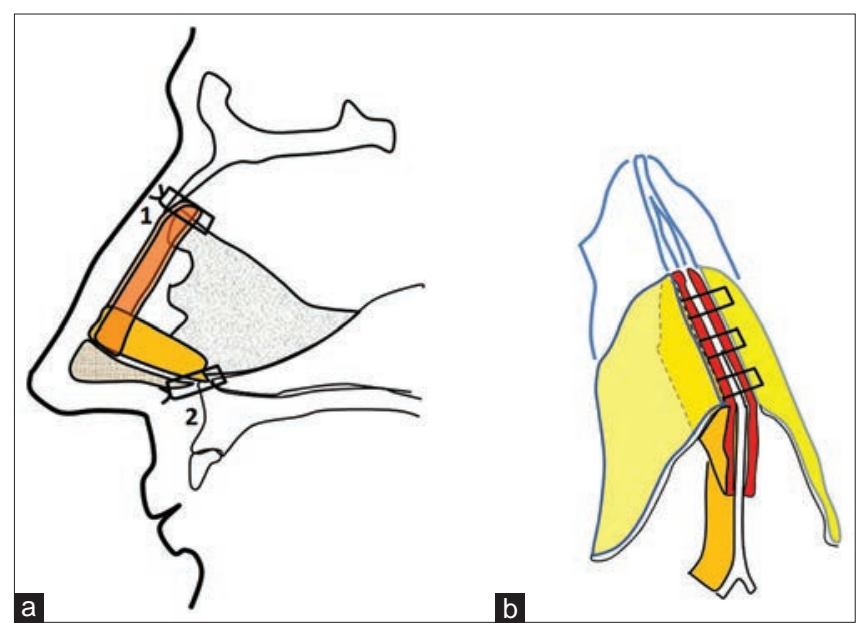

Figure 7: (a) The septal construct is sutured to the nasal bone (1) and anterior nasal spine below (2). (b) The lateral cartilages are sutured to anterior edge of the construct

were also done. Post-operative frontal view shows good correction with improved definition, lateral view shows better projection and a good profile line [Figure 9]. On worm's eye view, good tip support and definition is visible. The pliability of the lobule can also be appreciated [Figure 10].

\section{Patient 2}

A 20-year-old girl had a fall with the nose hitting the edge of the bed in childhood, resulting in the septal collapse. The septal component, the columellar strut and the dorsal onlay was constructed from the eighth costal cartilage. Osteotomies were done to improve the aesthetic appearance. She also required support grafts for upper lateral cartilages. These grafts were placed along the lower border of upper lateral cartilage reinforcing the internal valve [Figure 11]. Post-operative views show good tip configuration and mobility of the lobule is preserved [Figure 12]. The deformity is fully corrected; however, there is a fullness at the nasion indicating a graft size larger than required [Figure 13]. Although the patient was happy with the result, she was offered a revision with the possibility of further improvement. The revision of the same graft has produced an aesthetically pleasing result [Figure 14].

\section{Patient 3}

A 26-year-old man suffered from vehicular accident resulting in nasal septal collapse. Nose appeared broad with loss of tip projection. There was also apparent lengthening of the nose. Eighth costal cartilage was used for the septal component, columellar strut and dorsal onlay graft. Medial and lateral osteotomies were also done. Post-operative pictures show a good correction, 


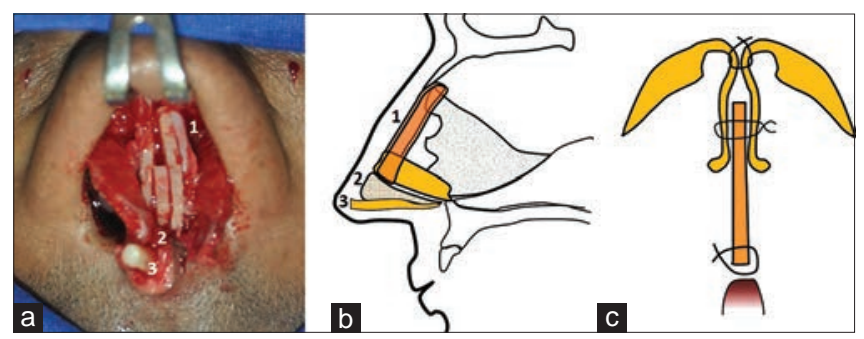

Figure 8: $(a$ and b) Complete assembly of framework place in the pocket. 1: Septal construct. 2: Space representing membranous septum. 3: Columellar strut. (c) Columellar strut is loosely sutured to the anterior nasal spine area and the medial crura of alar cartilages

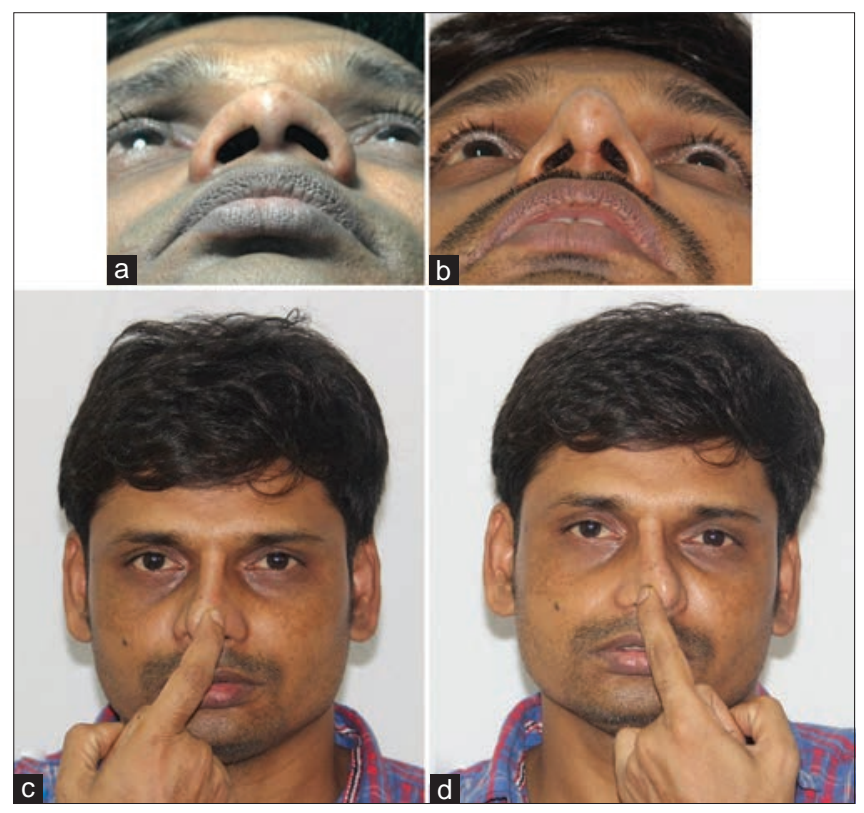

Figure 10: ( $a$ and $b)$ The basal view shows good tip support and configuration. (c and d) Demonstrates good pliability of the lobule

better dorsal projection [Figure 15] and definition and good tip support along with good mobility of lower one-third of the nose [Figure 16].

\section{DISCUSSION}

The nasal framework in upper third is formed by nasal bones laterally and perpendicular plate of ethmoid in the midline rendering it rigid and immobile. Middle third is formed by nasal cartilaginous septum and upper lateral cartilages allowing slight mobility. The lower third or the lobule is mobile. It is supported by the alar cartilages. The corresponding septal part is membranous allowing free mobility.

The dorsal (anterior) part and the caudal part of the cartilaginous septum are important structurally as well as functionally. Hence, Killian in $19^{\text {th }}$ century, advocated preservation of at least $1.5 \mathrm{~cm}$ strips on the dorsal and
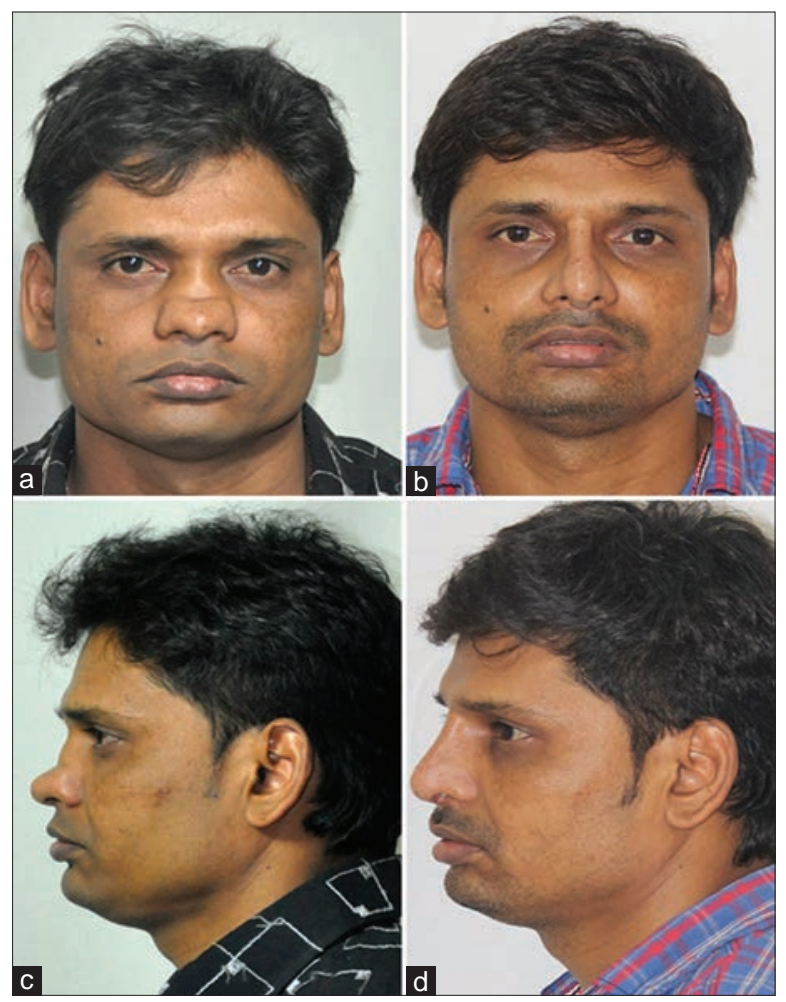

Figure 9: A 28-year-old man with typical deformity corrected with the three component framework. ( $a$ and $b$ ) Flattened preoperative appearance is corrected with good definition of dorsum. (c and d) The profile view shows adequate projection
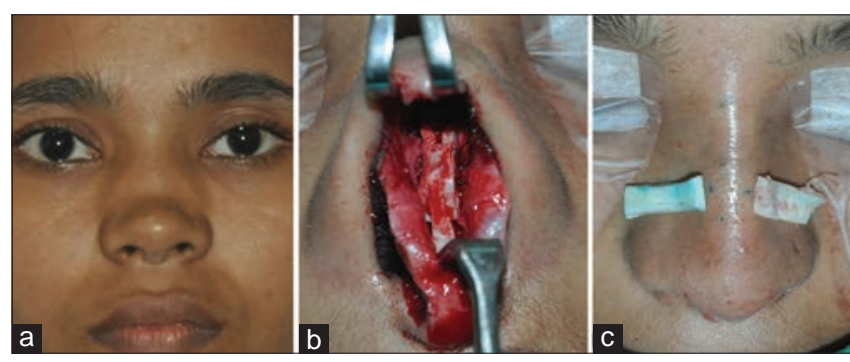

Figure 11: A 20-year-old girl with septal collapse. (a) Broad and flat nose in frontal view (b) The septal construct inserted into the pocket. (c) The surface marking of lateral cartilage support grafts which were placed internally along the lower border of lateral cartilage

caudal areas in an 'L' shape [Figure 17]..$^{[10]}$ Later on, it was understood that $1 \mathrm{~cm}$ width (instead of $1.5 \mathrm{~cm}$ ) of the dorsocaudal strut was enough to maintain the structural integrity. ${ }^{[10,11]}$ Rest of the bony and cartilaginous septum may be removed as a graft or remodelled. ${ }^{[1]}$ The present technique focuses on a recreation of these dorsal and caudal strips.

Nasal trauma might lead to septal framework collapse or resorption of septal cartilage secondary to haematoma or infection. ${ }^{[1]}$ Conventionally, septal collapse defects were treated with either cantilever technique or conventional 'L-graft' technique. Both these techniques ignore the 


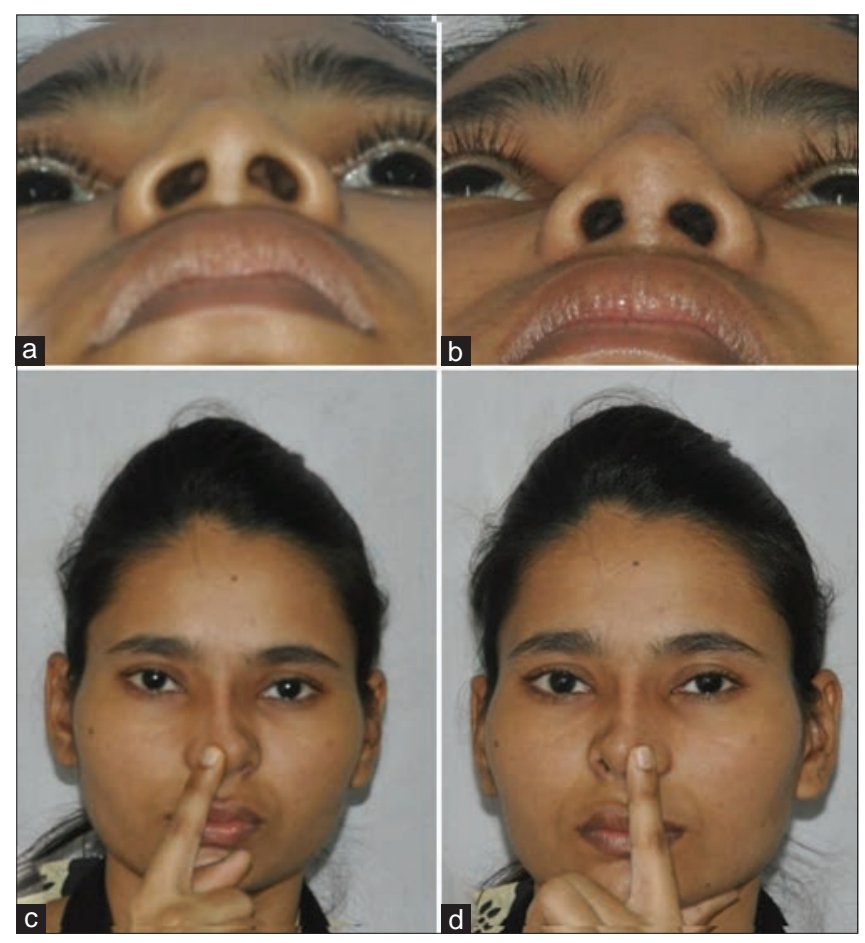

Figure 12: The result of the reconstruction (continued). ( $a$ and b). Good tip configuration. (c and d). Shows mobility of the lobule

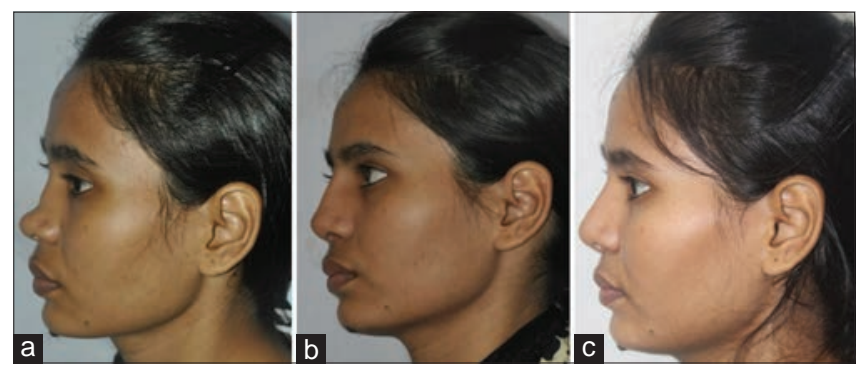

Figure 14: The revision procedure (a) The original deformity, (b) lateral view following first correction. (c) The appearance two and half months after revision of the graft. The prominence of the nasion area has reduced producing an aesthetically pleasing result

importance of critical part of the septum - the dorsal and caudal strips. In cantilever technique, the graft, either bony or cartilaginous, extends up to the tip with no caudal strut. It is fixed to the nasal bone with a stainless steel wire or a screw [Figure 18a]. The problem with this technique is that area around the hardware is under stress and may lead to loosening up of the framework and subsequent collapse. The bony part may get resorbed adding to the problem. ${ }^{[4]}$

Conventional 'L-graft' is formed from costal cartilage or bone and has dorsal and caudal limbs. The caudal limb lies in the columella and not in the caudal septal area. The dorsal limb extends from the radix to the tip of the nose [Figure 18b]. The disadvantage is that the lobule becomes rigid restricting the mobility of the tip

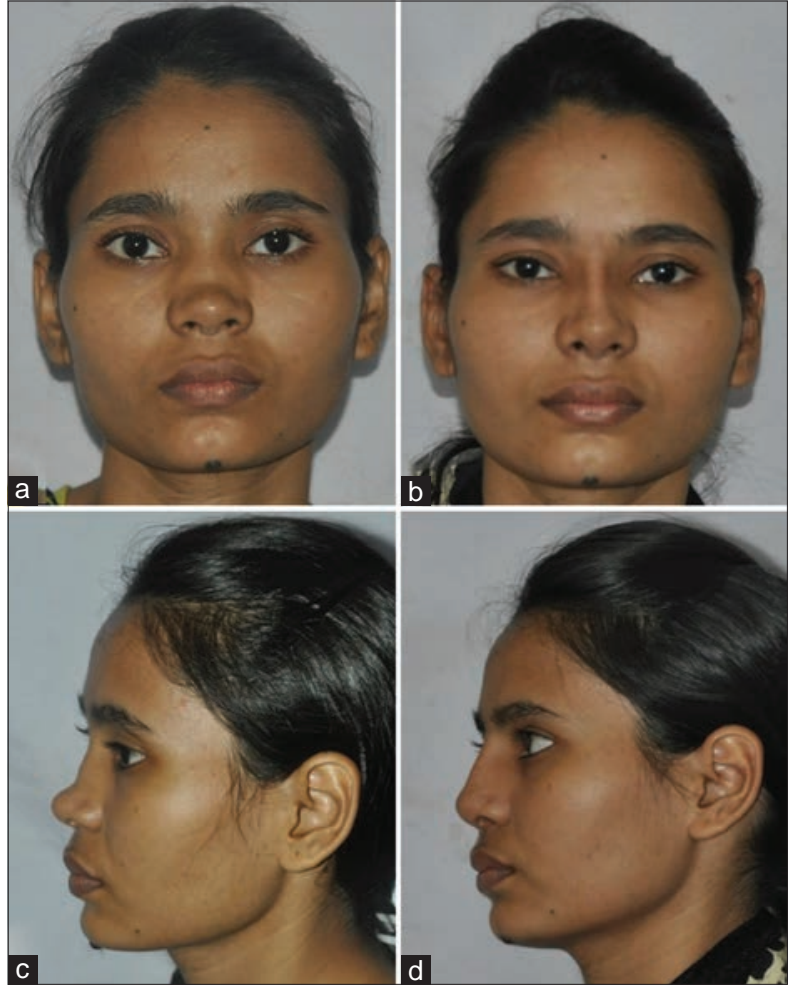

Figure 13: The result of reconstruction. ( $a$ and $b$ ). The frontal view shows good definition and a narrow slender nose. (c and d). In the lateral view- there is good projection of the dorsum

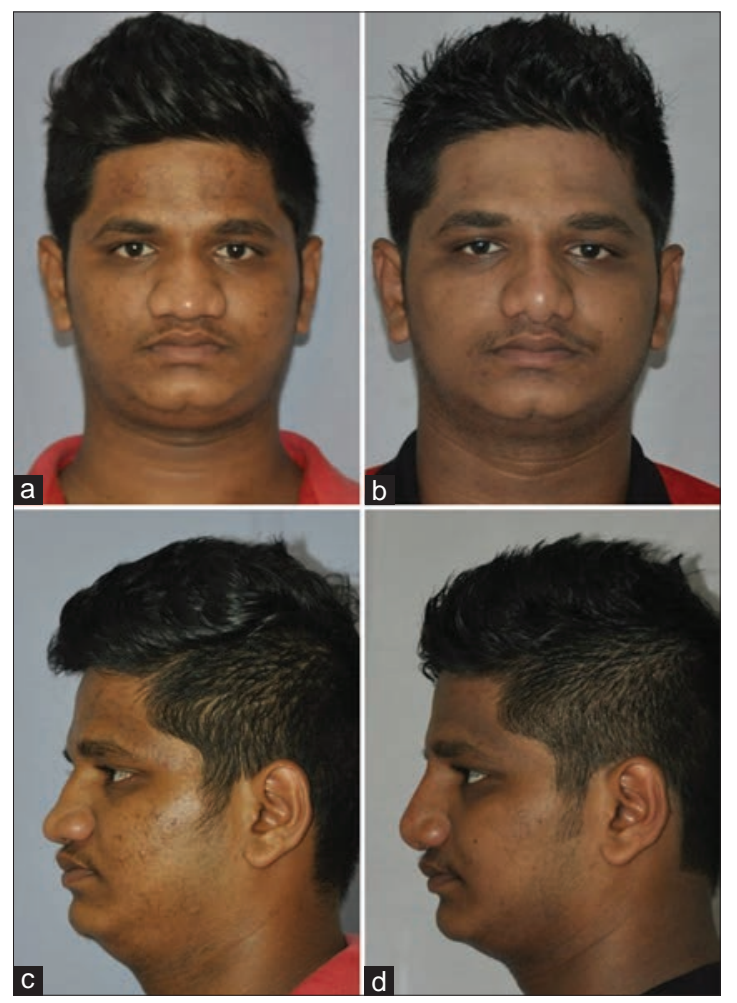

Figure 15: A 26-year-old man with broad and flat nose. The result shows good correction in frontal view ( $a$ and $b$ ) and in lateral view (c and d)

and during high-pressure activity at nose like sneezing, framework may buckle down. ${ }^{[1]}$ 

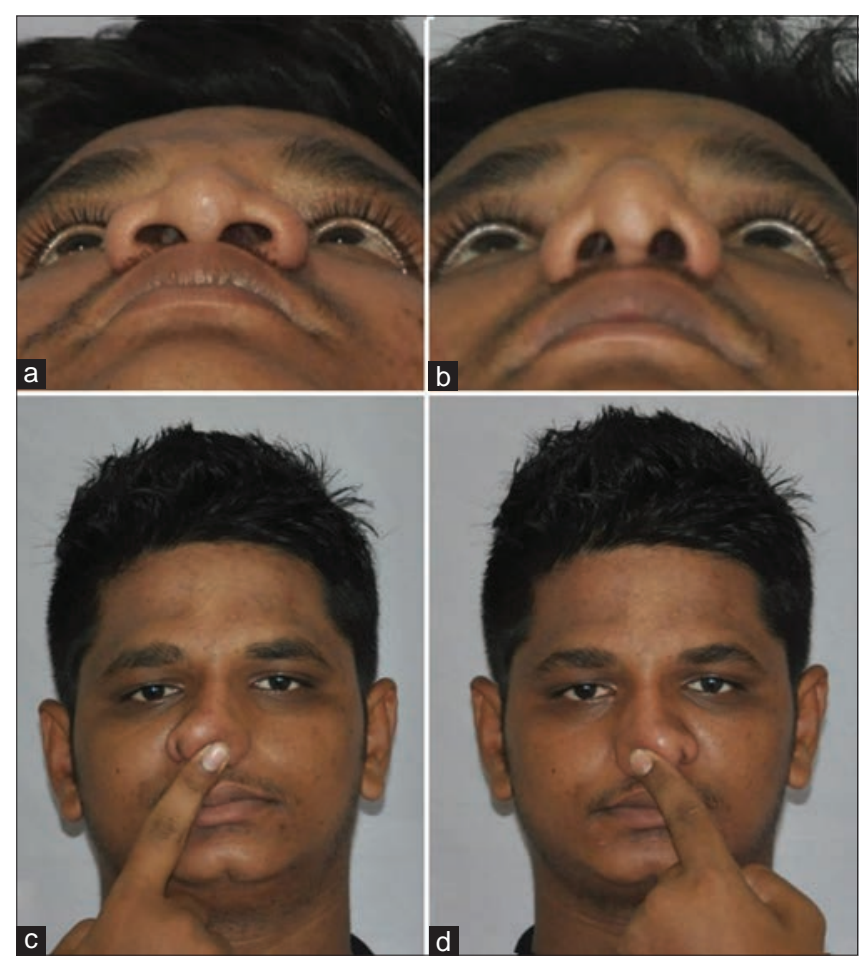

Figure 16: A good tip support in visible in the basal view ( $a$ and $b$ ). The mobility of the lobule is well appreciated (c and d)

The septal component of the present technique, although $\mathrm{L}$ shaped, is different from the conventional 'L graft'. The three-piece construct mimics the structurally important dorsocaudal part of the cartilaginous septum. The dorsal strips are thick enough to mimic the spreader grafts and provide support to the internal valve resulting in relief of airway obstruction. The dorsal component extends only till the septal angle area and not up to the tip. Unlike the conventional 'L graft', the caudal part of our framework represents the natural caudal area of the septum. It keeps the membranous septum free as it lies cranial to the membranous septum and not in the columella. Columellar support is given by a separate strut. This anatomical framework reconstruction [Figure 18c] maintains the pliability of membranous septum and lobule, unlike the rigid conventional 'L-graft'. As the septal and columellar supports are provided by two separate components, the lobule remains structurally separate from the upper two-thirds and is free to move.

Daniel $^{[6]}$ and Cakmak et al. ${ }^{[12]}$ have described their classifications of the saddle nose deformity based on the severity of the defect and the treatment offered. Our patients fall mainly in Type III of Daniel classification. A composite reconstruction is recommended for such defects with replacement of the dorsocaudal septal strut, a columellar strut and a diced cartilage fascia graft

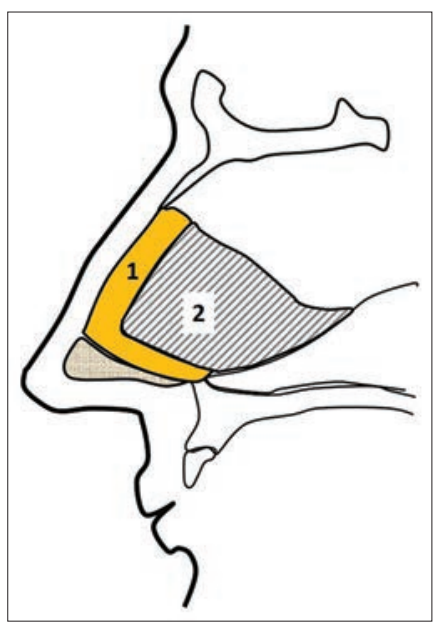

Figure 17: Structurally important part of the septum (1). The shaded area (2) can be safely removed

for the dorsum. However, our patients had additional features similar to Type IV. Although the bony vault is not disrupted, in the Indian context it is inherently small, necessitating a structural dorsal graft. Second, even if the vestibular lining is not contracted, the skin-soft tissue envelope is not as elastic. A tough skin envelope may exert a compression force on the underlying graft and a pliable graft may yield to it. Hence, we prefer a solid graft for dorsal onlay. We avoid resorption by not including any bony component in the costal graft, and it is shaped by precision carving technique to avoid distortion. ${ }^{[8]}$ We also feel that when the upper lateral cartilages do not have enough stiffness, grafts for upper lateral cartilage support may be required.

We believe the structural onlay graft is an essential component of the framework for two reasons. First, it provides support to the internal valve [Figure 18c]. ${ }^{[13,14]}$ Although the septal construct component is adequate for this purpose, the onlay graft provides the additional anterior framework support. Second, septal construct alone may not adequately meet the aesthetic demands of projection, definition and width of the nasal dorsum. In Indian patients, the aesthetic needs are different in that they generally need more projection. ${ }^{[15]}$ As the size and shape of the onlay graft can be tailored as per the requirements, it provides the ability to precisely control the dorsal projection, definition and width. The aesthetic needs are better met with using a third onlay component rather than using the septal component alone, and this should be the method of choice in Indian patients. In addition, maneuvers such as osteotomies and alar wedge resection may be required to enhance aesthetic and functional outcome. This technique has produced 


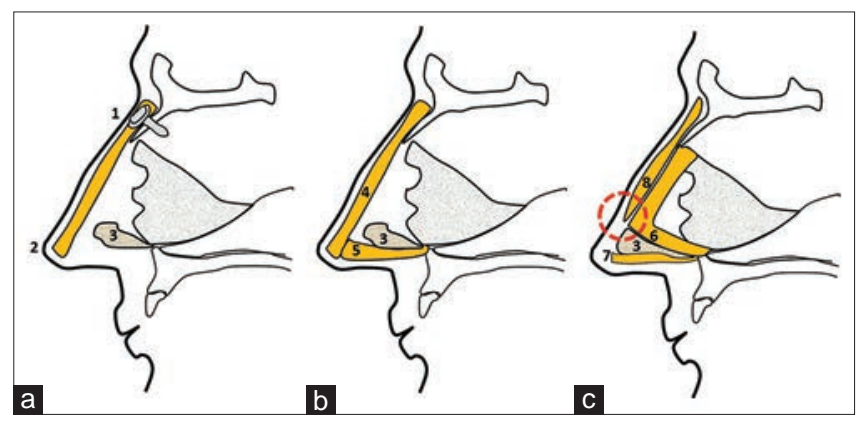

Figure 18: Comparison of techniques. (a) Cantilever technique - Dorsal bone graft is fixed to the nasal bones with a screw (1), extending till the tip (2).

Collapsed membranous septum (3). (b) Conventional L-graft technique- Dorsal graft extends to the tip (1), caudal limb (2) lies in the columella below the membranous septum (3). (c) Our technique- Septal construct's caudal piece (6) lies cranial to the membranous septum while the columella strut (7) lies below it. Dorsal onlay graft (8) ends at the septal angle area. Both the septal construct and dorsal onlay support the internal valve (circled) well

uniformly good results. One patient showed prominence of the graft at the nasion, however, this can be considered as error of judgement and not a failure of the technique. The revision of this graft was relatively easy, indicating versatility of the technique.

This technique has three distinct advantages over the traditional techniques:

i. It maintains pliability of the lobule as the framework is constructed in separate components

ii. It ensures better support to the internal valve as it incorporates three different ways of valve support: the septal construct that mimics spreader grafts, the dorsal onlay graft and the upper lateral cartilage support grafts, thus minimising the chances of failure

iii. The dorsal onlay component also offers better control of aesthetic needs.

\section{CONCLUSION}

Post-traumatic nasal septal collapse is a difficult entity to treat. Restoration of the natural anatomical framework commands not only support to the internal nasal valve, creation of separate stable and mobile nasal units but also shaping an aesthetically pleasing dorsum. Our technique satisfies these criteria improving the outcome.

\section{Declaration of patient consent}

The authors certify that they have obtained all appropriate patient consent forms. In the form the patient(s) has/have given his/her/their consent for his/her/their images and other clinical information to be reported in the journal. The patients understand that their names and initials will not be published and due efforts will be made to conceal their identity, but anonymity cannot be guaranteed.

\section{Financial support and sponsorship}

Nil.

\section{Conflicts of interest}

There are no conflicts of interest.

\section{REFERENCES}

1. Mull CC, Ginsburg MA. Drainage and packing of a nasal septal hematoma. Textbook of Pediatric Emergency Procedures. $2^{\text {nd }}$ ed. Ch. 51. Philadelphia: Lippincott Williams \& Wilkins; 2008. p. 615.

2. Chait LA, Becker H, Cort A. The versatile costal osteochondral graft in nasal reconstruction. Br J Plast Surg 1980;33:179.

3. Brown JB, McDowell F. Plastic Surgery of Nose. St. Lois, MO: C.V. Mosby Company; 1951.

4. Thaller SR, Bradley JP, Garri JI. Craniofacial Surgery. New York: Informa Healthcare; 2008. p. 59-81.

5. Patrick KS, Verma M, Arlene AR. Craniomaxillofacial Reconstructive and Corrective Bone Surgery. Ch. 44. New York: Springer; 2013. p. 483.

6. Daniel RK. Rhinoplasty: Septal saddle nose deformity and composite reconstruction. PlastReconstr Surg 2007;119:1029-43.

7. Constantian MB. Plastic and Reconstructive Surgery. Part 1. $2^{\text {nd }}$ ed. Vol. 2. Philadelphia: Saunders (W.B.) Co. Ltd.; 2005. p. 557.

8. Bhat U, Garg S, D'Souza EJ, Agarkhedkar N, Singh IA, Baliarsing AS, et al. Precision carving of costal cartilage graft for contour fill in aesthetic and reconstructive rhinoplasty. Indian $J$ Plast Surg 2014;47:25-35.

9. Guyuron B. Precision rhinoplasty. Part I: The role of life-size photographs and soft-tissue cephalometric analysis. Plast Reconstr Surg 1988;81:489-99.

10. Parrilla C, Artuso A, Gallus R, Galli J, Paludetti G. The role of septal surgery in cosmetic rhinoplasty. Acta Otorhinolaryngol Ital 2013;33:146-53.

11. André RF, Vuyk HD. Reconstruction of dorsal and/or caudal nasal septum deformities with septal battens or by septal replacement: An overview and comparison of techniques. Laryngoscope 2006;116:1668-73.

12. Cakmak O, Emre IE, Ozkurt FE. Identifying septal support reconstructions for saddle nose deformity: The Cakmak algorithm. JAMA Facial Plast Surg 2015;17:433-9.

13. Constantian MB, Clardy RB. The relative importance of septal and nasal valvular surgery in correcting airway obstruction in primary and secondary rhinoplasty. Plast Reconstr Surg 1996;98:38-54.

14. Daniel RK. Mastering Rhinoplasty: A Comprehensive Technique Atlas of Surgical Techniques with Integrated Video Clips. $2^{\text {nd }}$ ed. Ch. 6. Heidelberg: Springer-Verlag; 2010. p. 204.

15. Bhat U, Patel B. Primary rhinoplasty: An Indian perspective. Indian J Plast Surg 2008;41:S9-19. 